\title{
Comparative Study of Endoscopic Findings and CT-Para Nasal Sinuses appearances in Chronic Sinusitis
}

\author{
PV Sampath Kumar ${ }^{1}$, T. Rajendra Prasad ${ }^{2}$, K. Santhaiah ${ }^{3}$ \\ ${ }^{I}$ Dept of Otorhinolaryngology, Guntur Medical College, Guntur, India, 9440263426.
}

\begin{abstract}
Chronic sinusitis is one of the commonest causes of patients visit to the otorhinolaryngologist. Surgical clearance of these chronically infected sinuses while maintaining their ventilation and drainage is the treatment of choice. To achieve this goal, there should be some diagnostic modality, which guides us towards exact diagnosis and safe intervention. Over past decade, both CT and nasal endoscopy have been used successfully as diagnostic modality in sinus disease. To perform functional endoscopic sinus surgery effectively and safely, the surgeon must have detailed knowledge of the anatomy of the lateral nasal wall, Para nasal sinuses and surrounding vital structures. Sinus disease management has been technologically enhanced in recent years by improved radiographic evaluation and methods for intranasal visualization .Computerized Tomography (CT) provides essential pre-operative information for the assessment of patients undergoing functional endoscopic sinus surgery (FESS). One of the aims of CT of the sinuses is to delineate the extent of the disease, define any anatomical variants and relationship of the sinuses with the surrounding important structures. At present, CT scanning is the most used imaging technique for assessing chronic sinusitis and defining the anatomic abnormality. The primary role of the coronal CT scan is to determine the extent and possibly the underlying cause of sinusitis. As a rule, surgeons individualize their surgical approach according to the amount and location of disease they see on CT scan.

Endoscopic techniques for Para nasal sinus surgery have allowed detailed and complete visualization of sinus disease while promising minimum distress to the patient. The telescopic view of the operative field shows detail of the sinus anatomy and its disease. It has been possible to see areas of the cribriform and orbital wall that are at risk to produce cerebrospinal fluid rhinorrhea and orbital complications during the surgery. At the same time, landmarks for avoiding these complications can be defined to guide the surgeon during the surgery as seen through the endoscope. Recently combination of diagnostic endoscopy and systematic understanding of the lateral nasal wall with CT in the coronal plane has become the corner stone in the evaluation of the PNS disease. This is the basis of the new concept of FESS.

In our study we have compared the diagnostic endoscopic and CT findings with the operative findings of patients with sinus diseases.
\end{abstract}

Keywords: CT: Computed tomography, FESS: Functional Endoscopic Sinus Surgery, PNS: Para Nasal Sinuses, DNE: Diagnostic Nasal Endoscopy.

\section{Introduction}

Various methods for evaluation of chronic sinusitis are Diagnostic Nasal Endoscopy, CT Scan of PNS and MRI of PNS. CT of PNS has achieved wide clinical use over the past many years.

\section{Diagnostic Nasal Endoscopy (DNE)}

It is a simple office procedure, done under local anesthesia. Hopkin's rod optical system with cold light source and fibre optic light delivery system provides an excellent illumination and optical quality. Along with CT scan this gives complementary information regarding extent of disease and anatomical and pathological variations.

CT scan Imaging Of Nasal Cavity And Paranasal Sinuses: CT scanning of the paranasal sinus disease proves by far to be the most reliable method of pre-operative assessment. It has the advantage of showing both bony details, using wide window settings, and good soft tissue outline, using narrow window settings. Both axial and coronal views may be of use - although coronal views are more helpful.

\section{Material And Methods}

This present study entitled "A Comparative Study of Endoscopic Findings and CT-Para nasal Sinuses Appearances in Chronic Sinusitis”. A prospective study of sinus diseases using diagnostic nasal endoscopy and 
computed tomography was conducted in the Department of ENT, Government General Hospital, Guntur. A total of 50 patients who had chronic sinusitis for more than three months duration not responding to the medical line of treatment and who were willing to undergo Functional Endoscopic Sinus Surgery are subjected to undergo DNE and CT of PNS.

The Equipments used are Nasal endoscope: Karl Storz Hopkins rod optical with cold light source and fiber optic light delivery system. Endoscopes used were with $0^{\circ}, 30^{\circ}$ and $70^{\circ}$ angles of view of $4 \mathrm{~mm}$ diameters.

Karl Storz Endo vision Telecom deluxe camera system with monitor.

\section{Sample Size: 50}

Sampling: Prospective Study.

Inclusion Criteria: All the patients with clinically proven chronic sinusitis not responding to routine medical line of treatment.

Major Factors: Facial pain/pressure, nasal obstruction, nasal discharge/discolored postnasal drip, hyposmia/anosmia, purulent secretions on examination, and fever (only in acute sinusitis).

Minor factors: Headache, fever, halitosis, dental pain, fatigue, cough, and ear pain/pressure/fullness.

Concurrent signs of sinonasal inflammation must be present on CT scan.

Exclusion Criteria: Patients with acute attack of sinusitis, Patient with sinus malignancies, Patient who were not willing to undergo FESS and Fungal Sinusitis.

\section{Results}

Statistical Analysis: The present study was conducted on 50 patients in the Department of ENT, Govt. General Hospital, Guntur. Our study included fifty patients, out of which bilateral surgery was done in 33 patients and unilateral was done on 17 patients. Middle meatal antrostomy, infundibulotomy along with anterior ethmoidectomy and frontal recess clearance was done in all the cases. While polypectomy, posterior ethmoid clearance and sphenoidotomy was done in some cases.

Age Distribution: The age of the patient in our study varied from $21 \mathrm{yrs}$ to 55yrs. Maximum number of patients were in 31 to 35 years of age group, therefore $34 \%$ of patients were in early $3^{\text {rd }}$ decade of age.

Table 1-Age distribution

\begin{tabular}{|c|c|c|}
\hline Age & No of Patients & Percentage \\
\hline $21-25$ & 3 & 6 \\
\hline $26-30$ & 10 & 20 \\
\hline $31-35$ & 17 & 34 \\
\hline $36-40$ & 10 & 20 \\
\hline $41-45$ & 5 & 10 \\
\hline $46-50$ & 3 & 6 \\
\hline $51-55$ & 2 & 4 \\
\hline Total & 50 & 100 \\
\hline
\end{tabular}




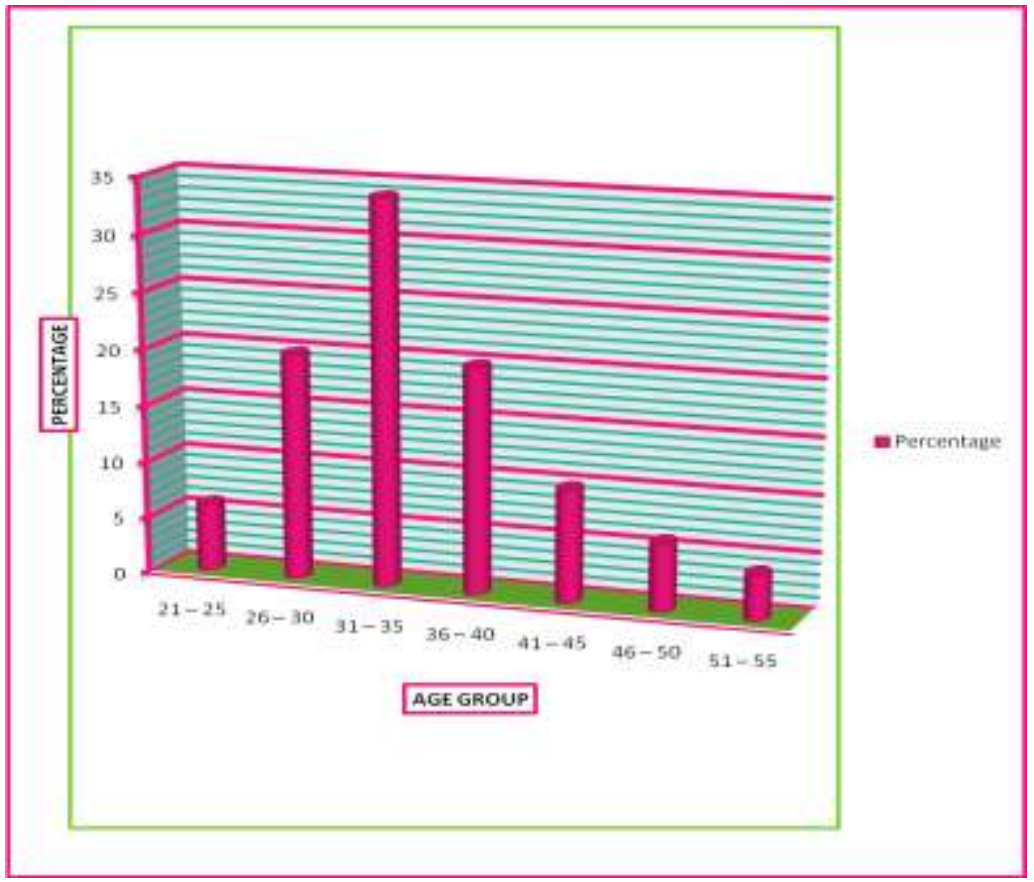

Sex distribution: Our study showed male preponderance i.e. $64 \%$ male and $36 \%$ female patients. Thus male to female ratio was 1.8:1.

Table - 2 Sex Distribution

\begin{tabular}{|c|c|c|}
\hline Sex & No of Patients & Percentage \\
\hline Male & 32 & 64 \\
\hline Female & 18 & 36 \\
\hline Total & 50 & 100 \\
\hline
\end{tabular}

Sexdistribution:

\section{Percentage}

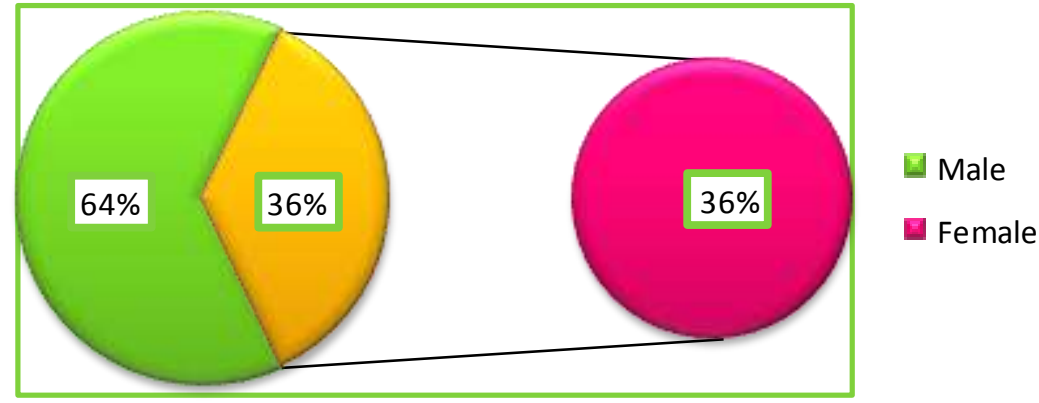


Symptoms: Common symptoms of the patients in our study were headache, nasal discharge and nasal obstruction, sneezing and hawking were less common. In our study $84 \%$ patients had headache, $84 \%$ had nasal obstruction, $70 \%$ had nasal discharge, postnasal discharge was seen in $44 \%$ of patients and sneezing in $30 \%$.

Table 3- Distribution of symptoms in patients with chronic sinusitis

\begin{tabular}{|c|c|c|}
\hline Stmptoms & Joof Patents & Peprentitgeg \\
\hline NanlObandition & 42 & $8 t$ \\
\hline Nagl Discharge & 35 & 70 \\
\hline Headarla Facial Paill & 42 & 84 \\
\hline Post.Mal Lischarge & 21 & H \\
\hline Shering & 15 & 30 \\
\hline Others & 3 & 6 \\
\hline
\end{tabular}

Distribution of percentage of symptoms in chronic sinusitis:

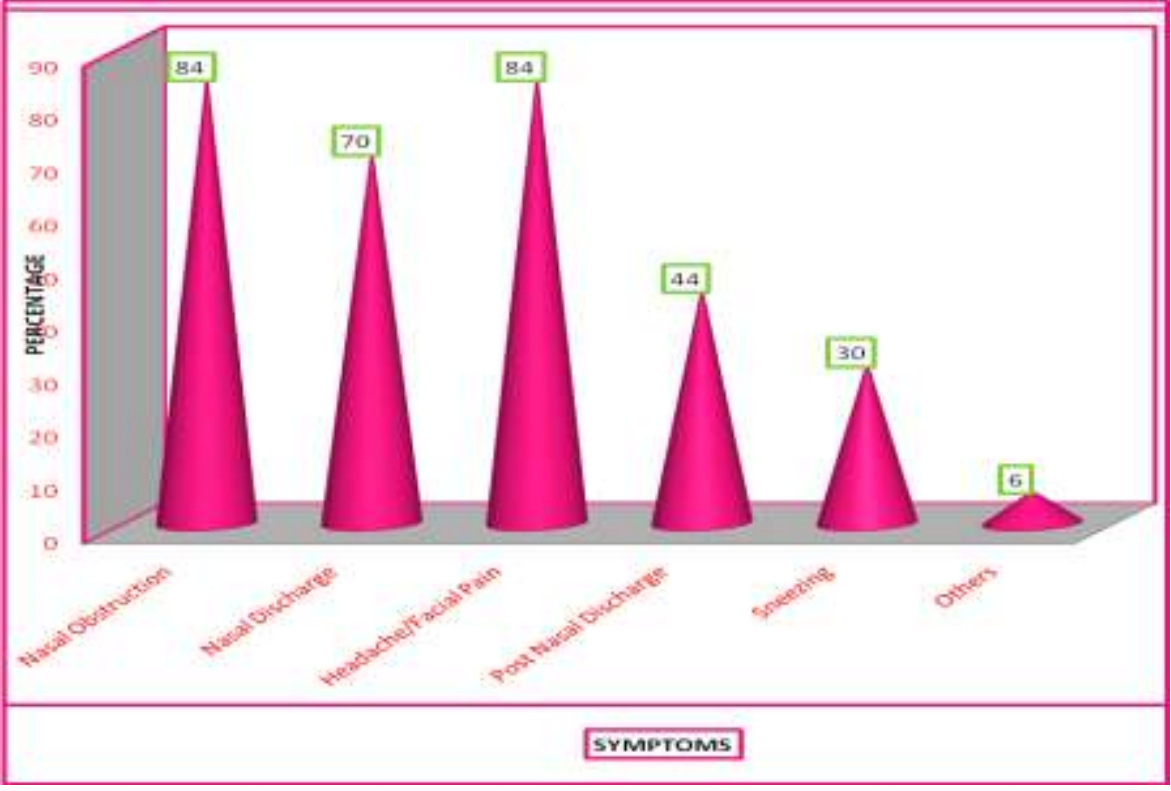


Signs: In our study, commonest sign was edematous nasal mucosa in $36 \%$ patients; sinus tenderness was seen in $34 \%$ of patients followed by purulent middle meatal discharge in $24 \%$ and nasal polyps in $30 \%$ of patients. Hypertrophied middle turbinate was found in $20 \%$ whereas, purulent middle meatal discharge was seen in $30 \%$ of the patients

Table -4 Distribution of signs in patients with chronic sinusitis

\begin{tabular}{|c|c|c|}
\hline Signs & No of Patients & Percentage \\
\hline Nasal Mucosa: Congested & 8 & 16 \\
\hline Nasal Mucosa: Pale & 13 & 26 \\
\hline Nasal Mucosa: Edematous & 17 & 36 \\
\hline Inferior Turbinate Hypertrophy & 8 & 20 \\
\hline Middle Turbinate Hypertrophy & 10 & 30 \\
\hline Middle Meatus: Non Purulent & 15 & 24 \\
\hline Middle Meatus: Purulent & 12 & 30 \\
\hline Nasal Polyps & 15 & 34 \\
\hline Sinus Tenderness & 18 & 44 \\
\hline Others & 22 & \\
\hline
\end{tabular}


Diagnosis: $74 \%$ of the patients in our study suffered from chronic sinusitis whereas $26 \%$ presented with bilateral nasal polyposis.

Table - 5 Diagnosis of patients in our study

\begin{tabular}{|c|c|c|}
\hline Diagnosis & No of Patients & Percentage \\
\hline Chronic Sinusitis & 37 & 74 \\
\hline Gross Nasal Polyposis & 13 & 26 \\
\hline Total & 50 & 100 \\
\hline
\end{tabular}

Endoscopic operative procedures performed: Before undergoing functional endoscopic sinus surgery every patient underwent thorough evaluation of nose and par nasal sinus through diagnostic nasal endoscopy and computerized tomography and the results were confirmed during the operative procedure. The various operative procedures performed in our study are as follows. Out of fifty cases, thirty-three underwent bilateral procedures where as seventeen underwent unilateral procedure. Septoplasty was done in 6 patients. Frontal recess clearance was done in 20 patients, 13 patients underwent ethmoidectomy, 50 patients underwent middle meatal antrostomy and 16 patients underwent sphenoidotomy.

\section{Operative procedure}

Table - 6 Operative procedures

\begin{tabular}{|l|l|}
\hline Septoplasty & 06 \\
\hline Frontal Recess Clerance & 20 \\
\hline Ethmoidectomy & 13 \\
\hline Middle Meatal Antrostomy & 50 \\
\hline Sphenoidotomy & 16 \\
\hline
\end{tabular}

\section{Correlation of diagnostic endoscopy findings with computed tomography findings:}

The parameters correlated in our study include middle turbinate, middle meatus, bulla ethmoidalis, hiatus semilunaris, forntal recess and sphenoethmoid recess. The sensitivity and specificity were calculated for diagnostic endoscopy as compared to CT findings. Diagnostic endoscopy was found to have sensitivities for frontal recess, hiatus semilunaris and sphenoethmoidal recess as $90 \%, 94 \%$ and $100 \%$ respectively. While sensitivity for middle turbinate, bulla ethmoidalis and middle meatus was $74 \%, 62 \%$ and $85 \%$ respectively. The sensitivity of diagnostic endoscopy for frontal recess, middle turbinate and bulla ethmoidalis was found as $91 \%$, $84 \%$ and $76 \%$ respectively and for sphenoethmoidal recess, hiatus semilunaris and middle turbinate as $80 \%$, $53 \%$ and $67 \%$ respectively.

\section{Correlation of CT findings with Operative findings:}

The various parameters correlated were inferior turbinate, middle meatus, middle turbinate, uncinate process, shenoethmoid recess, frontal recess, frontal sinus, maxillary sinus, ethmoid sinus, sphenoid sinus and septum. The false positive, false negative, sensitivity and specificity of CT as compared to operative were calculated for each parameter. CT showed sensitivity for maxillary sinus, posterior ethmoids, haller cells and uncinate process which were $98 \%, 92 \%, 100 \%$ and $92 \%$ respectively. CT was found to be relatively less sensitive for parameters like infundibulum, hiatus semilunaris, frontal recess and middle meatus. The values were $60 \%, 77 \%, 88 \%$ and $89 \%$ respectively. The specificity of CT was found to be best for hiatus semilunaris, haller cells, frontal recess, inferior meatus, anterior ethmoids, posterior ethmoids and inferior turbinate. The values were $90 \%, 100 \%, 94 \%, 97 \%, 90 \%, 98 \%$ and $96 \%$ respectively. CT has relatively less specificity for bulla ethmoidalis, maxillary sinus, middle meatus and sphenoethmoid recess. The values were $74 \%, 84 \%, 83 \%$ and $86 \%$ respectively.

\section{Correlation of diagnostic endoscopy findings with operative findings:}

The various parameters correlated were inferior turbinate, middle meatus, middle turbinate, uncinate process, sphenoethmoid recess, frontal recess, frontal sinus, maxillary sinus, ethmoid sinus, sphenoid sinus and septum. In our study, the sensitivity of diagnostic nasal endoscopy was more for middle meatus, uncinate process, hiatus semilunaris, bulla ethmoidalis and ager nasi cell. The values were $93 \%, 92 \%, 100 \%, 91 \%$ and 95\% respectively. The diagnostic nasal endoscopy sensitivity was comparatively less for sphenoethmoid recess, frontal recess, inferior meatus and inferior turbinate. The values were $75 \%, 83 \%, 89 \%$ and $84 \%$ respectively. The specificity of diagnostic nasal endoscopy was more for hiatus semilunaris, frontal recess, ager nasi cell, inferior meatus and inferior turbinate. The values were 100\%, 93\%, 96\%, 93\% and 92\% respectively. The specificity of diagnostic nasal endoscopy was comparatively less for middle meatus, uncinate process, bulla 
ethmoidalis, sphenoethmoid recess and middle turbinate. The values were $79 \%, 79 \%, 85 \%, 83 \%$ and $88 \%$ respectively.

Correlation of diagnostic nasal endoscopic, Computed Tomographic and operative findings: In our study the diagnostic endoscopic findings and CT findings correlated very well with the operative findings, keeping in view that $\mathrm{CT}$ is better than endoscopy in most of the parameters except for uncinate process, sphenoethmoid recess and middle meatus.

\section{Discussion}

The present study entitled "A Comparative Study of Endoscopic Findings and CT-Paranasal Sinuses appearances in Chronic Sinusitis" was conducted in the Department of ENT, Govt. General Hospital, Guntur from September 2013 to August 2015. The study included 50 patients of chronic sinusitis who did not respond to medical line of treatment and are willing to undergo Functional Endoscopic Sinus Surgery. Out of the 50 patients 33 underwent bilateral and 17 underwent unilateral endoscopic surgeries. All the cases had undergone diagnostic endoscopy and computed tomography before the operation.

In our study age of patients varied between 21 and 55 years with the maximum number of patients in 31 to 35 year age group. In the present study 32 patients i.e $64 \%$ were males while 18 patients i.e. $36 \%$ were females. In our study, nasal obstruction and headache were the commonest symptoms both of which are present in 42 patients. The next frequently occurring complaint was nasal discharge present in 35 patients. The other symptoms that were noted are postnasal discharge in 22 patients, sneezing in 15 patients and other symptoms like fever, epistaxis, anosmia/cacosmia etc in 3 patients. In the majority of patients, the duration of symptoms were for more than 6 months. In our study, the commonest clinical signs present were nasal mucosal oedema in 17 patients and congested mucosa in 8 patients, while a pale mucosa was present in 13 patients. The other findings were nasal polyps in 15 patient's, middle meatal discharge-mucopurulent in 15 patients and nonpurulent in 15 patients and sinus tenderness in 18 patients. Middle turbinate hypertrophy was seen in 10 patients. In the present study 37 patients $(74 \%)$ had chronic rhino sinusitis, while 13 patients $(26 \%)$ had gross sinonasal polyposis.

All the patients included in our study underwent diagnostic nasal endoscopy followed by CT scan. On endoscopy, in addition to gross findings such as pathologic discharge, subtle evidence of disease in the osteomeatal area was identified. Among the parameters that were correlated, the diagnostic endoscopy was found to be most sensitive investigation for the sphenoethmoid recess, middle turbinate and middle meatal area with sensitivity of $93.75 \%, 100 \%$ and $90 \%$ respectively, while the specificity was $53.19 \%, 80 \%$ and $91.71 \%$. However, the sensitivity and specificity for the middle turbinate $(74.5 \%$ and $84.4 \%)$ and middle meatus $(85.18 \%$ and $67.5 \%$ ) were not so good as compared to the above parameters. There were a significant percentage of various parameters that could not be visualized at diagnostic endoscopy are: middle turbinate, middle meatus, bulla ethmoidalis, hiatus semilunaris, frontal recess and sphenoethmoid recess. This is because in some of the cases it was impossible to pass the endoscope beyond certain point either due to presence of gross pathology like extensive polyposis or due to severe anatomical abnormalities like a severely deviated nasal septum, paradoxical middle turbincate, or a concha bullosa. CT scan definitely proved to be very helpful in these cases.

\section{CT PNS - Coronal section showing right DNS with Left concha bullosa.}

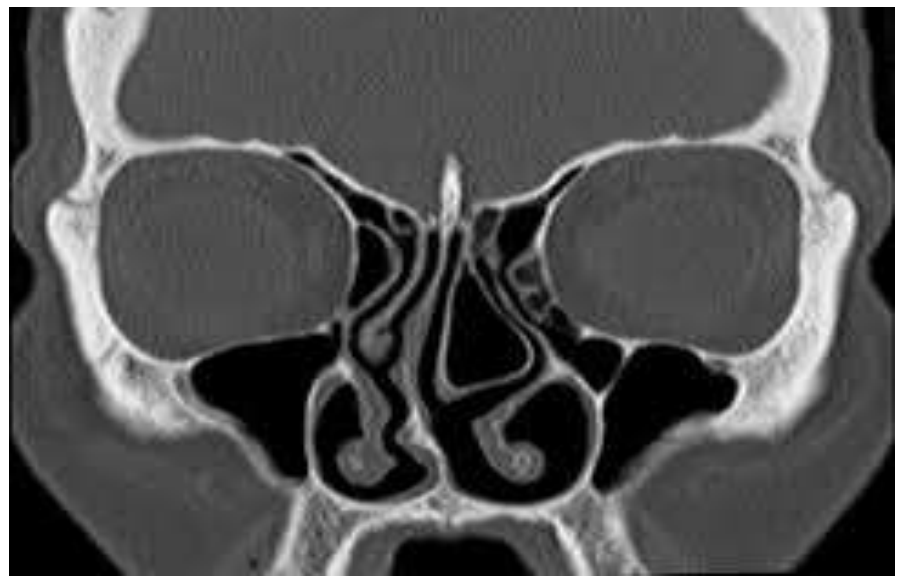


In the present study out of 50 patients, 33 underwent bilateral surgeries. Apart from that, 6 underwent septoplasty and 27 procedures of polypectomy were done along with FESS at the same time. 13 were ethmoidectomy, 20 were frontal recess clearance, 75 were middle meatal antrostomies, and 16 were sphenoidotomy.

$\mathrm{CT}$ is effective in demonstrating predisposing causes of chronic sinusitis (eg. anatomical variants) and provides precise guidance for therapeutic endoscopic instrumentation. CT with its excellent capability for displaying bone and soft tissues is the current diagnostic modality of choice for evaluating the osteomeatal complex. Among the various parameters that were correlated, the sensitivity was found to be good for almost all the parameters [maxillary sinus $(94.3 \%)$ frontal recess $(88 \%)$, and for the ethmoids $(88 \%)$ ]. The specificity of CT scan was found to be good for the ethmoids $(90.38 \%)$ and sphenoid sinus $(92.3 \%)$ than for the maxillary sinus $(84.2 \%)$.

\section{CT SCAN Coronal section showing bilateral maxillary mucosal thickening}

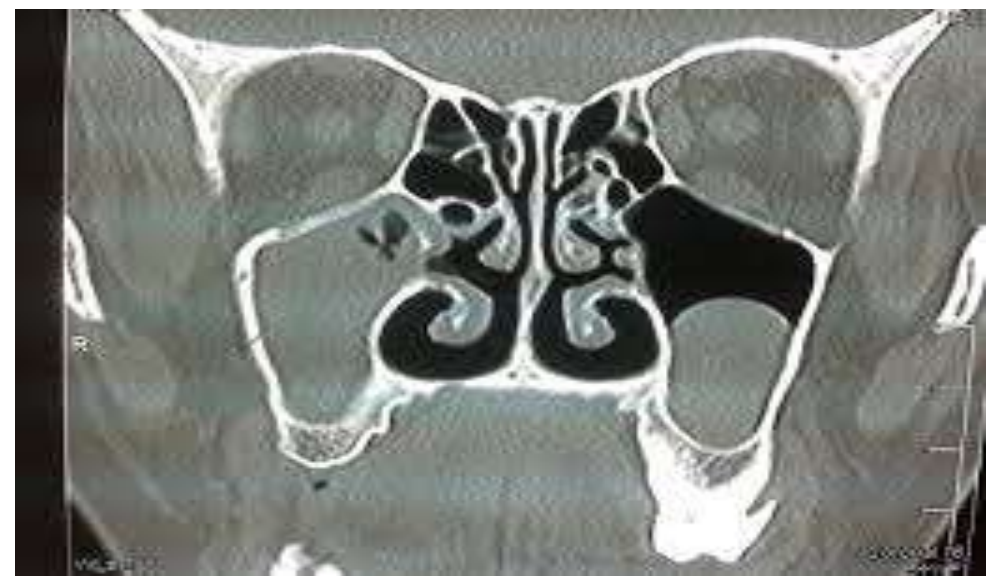

The CT scan serves as a "road map" for the surgeon as he negotiates the potentially hazardous clefts of the para nasal sinus unit. The diagnostic endoscopy findings correlated very well with the operative findings. But, the drawback of diagnostic endoscopy was that a significant proportion of cases in whom not all the parameters could be visualized due to presence of gross pathology or severe anatomical abnormality that made it impossible to pass the endoscope beyond certain point. Almost all the diagnostic endoscopic findings were confirmed at operation. The association between Diagnostic endoscopy and CT was calculated using Chi square test.

$$
\text { Chi square } \chi 2=77.3
$$

$$
\mathrm{P} \text { Value } \mathrm{P}=1 \mathrm{E}-11
$$

Since $\mathrm{P}=1 \mathrm{E}-11 .<0.05$ indicates there is a high association between diagnostic endoscopy and CT. Hence, the advantages of diagnostic endoscopy are Optical brilliance, clear field of vision, easy handling, office procedure, economic, ability to "look around corners" with deflecting angles, documentation and teaching purpose. Limitations of nasal endoscopy are gross septal deviation can make endoscopy difficult, localized disease within the infundibulum, frontal recess and maxillary sinus ostium is difficult to diagnose and extent of disease within the spheno-ethmoid is difficult to be made out. CT imaging of PNS can overcome these limitations. The computed tomographic findings correlated well with the confirmed findings at operation. The coronal section CT scans provided most of the information required for an endoscopic clearance. Advantages of CT scan are it shows progressively deeper structures as the surgeon encounters them during operation (eg: uncinate process, bulla ethmoidalis, ground lamella, sphenoid sinus, in an A-P direction), the relationships of the above structures to important areas such as the lamina papyracea and skull bone, reducing the morbidity, dehiscence's of the lamina papyracea are better visualized. To summarize, the CT scan serves as a "road map" for the surgeon as he negotiates the potentially hazardous clefts of the PNS unit. It is a non-invasive, rapid, convenient investigation, which helps in documentation and education. As already mentioned CT scan delineates the extent of disease, anatomical and pathological variations far better than other methods.

Disadvantages of CT scan was radiation dose to the sensitive areas like cornea and lens is particularly high when axial cuts are taken - nearly 185 times more than that recorded for plain $\mathrm{X}$ - rays. Careful positioning of the patient in the scanner can reduce this. Inability to differentiate fibrous tissue and inflammatory mucosal disease and it is a relatively expensive investigation. CT scan should be used to provide supplementary clinical data to the history and assist in directing surgical treatment to the affected areas. 


\section{Conclusion And Summary}

This study was conducted in the Department of ENT, Government General Hospital, Guntur from September 2013 to August 2015.This study was undertaken with the objective of correlating the diagnostic endoscopic and computed tomographic findings in patients with chronic sinusitis. Fifty patients suffering from chronic sinusitis underwent Functional Endoscopic Sinus Surgery after being thoroughly evaluated by diagnostic endoscopy and CT scan. The factors affecting the drainage of various sinuses along with the state of various sinuses were compared as per diagnostic endoscopy, computed tomography and operation. Sensitivity and specificity were calculated for both diagnostic endoscopy and computed tomography. Association were calculated using Chi square test with overall $\mathrm{P}$ value less than 0.05 signifying that there is a high association between both CT and endoscopy.

In the overall assessment, following conclusions can be drawn, maxillary sinus was most frequently diseased sinus in our study. Computed tomography imaging of PNS is an important preoperative evaluation tools in detecting pathology. The findings of CT PNS correlated very well with the operative findings except for very few findings. Extent of disease in each sinus was very well recognized in CT, when compared to diagnostic endoscopy. CT scan is a must prior to any functional endoscopic sinus surgery. It helps in assessing the extent of sinus disease and to know the variations and vital relations of the par nasal sinuses. CT assists the surgeon as a "road-map" during FESS.

\section{Acknowledgements}

Our sincere thanks to Head of the Radiology dept.: Professor Parvatheswara Rao.

\section{References}

[1]. Amit.nd dwivedi, kapil kumar singh: ct of the paranasal sinuses: normal anatomy, variants and pathology. Journal of Optoelectronics and Biomedical Materials Vol.2 Issue 4, October-December 2010, p. 281 - 289

[2]. Benninger MS, Holzer SE, Lau J. Diagnosis and treatment of uncomplicated acute bacterial rhinosinusitis: Summary of the Agency for Health Care Policy and Research evidence-based report. Otolaryngol Head Neck Surg 2000; 122: 1-7.

[3]. K dua, h chopra, as khurana, m munjal: CT Scan Variations in Chronic Sinusitis. Ind J RadiolImag 2005 15:3:315-320

[4]. Mark May MD, Steven M. Sobol MD, , KennethKorzec MD: The location of the maxillary Os and its importance to the endoscopic sinus surgeon. The Laryngoscope Volume 100, Issue 10, pages 1037-1042, October 1990.

[5]. Freitas MR, Vasconcelos DN, Freitas AE, Maia Filho JH, Castro e Silva Cd. Nasal endoscopic and CT scan alterations of the paranasal sinuses as predictors of severity in patients with cystic fibrosis. Braz Otorhinolaryngol. 2013 Aug;79(4):480-6

[6]. Rontal M, Rontal E.: Studying whole-mounted sections of the paranasal sinuses to understand the complications of endoscopic sinus surgery. Laryngoscope. 1991 Apr;101(4 Pt 1):361-6.

[7]. William E. Bolger MD,David S. Parsons , , FACS,Clifford A. Butzin: Paranasal sinus bony anatomic variations and mucosal abnormalities: CT analysis for endoscopic sinus surgery. The Laryngoscope Volume 101, Issue 1, pages 56-64, January 1991

[8]. Josef Shargorodsky MD, and Neil Bhattacharyya MD, What is the role of nasal endoscopy in the diagnosis of chronic rhinosinusitis The Laryngoscope January 2013 Volume 123, Issue 1, pages 4-6.

[9]. Stammberger H, Posawetz W.: Functional endoscopic sinus surgery. Concept, indications and results of the Messerklinger technique. Eur Arch Otorhinolaryngol. 1990;247(2):63-76.

[10]. Scott - Brown's Otolaryngology, Head and neck surgery, $7^{\text {th }}$ edition, vol 2 ,chapter $104, \operatorname{pg} 1315-1319$.

[11]. StambergerH, Wolf G.Headaches and sinus disease: the endoscopicapproach. Ann. Otol. Rhinol. Laryngol1988;34;2-23.

[12]. KaluskarSK,PatilNP.Value of CT in the evalution of chronic sinus disease. Ind JLO Head and Neck surg 1992;4:188-192

[13]. Kirtane MV et. al.Functional endoscopic sinus surgery (A preliminarystudy).Indian Journal of otolaryngology1991;43:126-9. 